\title{
LEVANTAMENTO DOS TIPOS DO HERBÁRIO DO JARDIM BOTÂNICO DO RIO DE JANEIRO: MELIACEAE I
}

\author{
ELSIE FRANKLIN GUIMARÃES * \\ LUCIANA MAUTONE * * \\ VALÉRIO FLECHTMANN FERREIRA *** \\ GUSTAVO MARTINELLI **
}

\section{SINOPSE}

Com este trabalho damos continuidade à série de outros que se vem realizando neste Jardim sobre Tipos do Herbácio do Jardim Botânico do Rio de Janeiro (RB), e sua classificação. É ilustrado com fotografias das espécies cujo material foi citado pelo autor.

\section{INTRODUÇÃo}

$\mathrm{Na}$ elaboração do presente trabalho seguimos os critérios abaixo relacionados por autores anteriores IOCCHIONI 1949, 1952, 1953), GUIMARÃES (1965, 1966), TRAVASSOS (1965, 1966), MENDES MARQUES (1976), RIBEIRO DE SOUZA e BENEVIDES (1977) que se ocuparam do levantamento de Tipos, principalmente do Herbário do Jardim Botânico do Rio de Janeiro.
a) Citação da espécie
b) Citação do autor e da obra Origina!
c) Transcrição do material examinado (Tipo), tal como citado na obra Original.
d) Citação da sigla do Herbário do Jardim Botânico, seguida do número de registro.
e) Classificação do Tipo
f) Transcrição das diversas etiquetas (schedulae) encontradas nas excicatas.
g) Fotografias dos Tipos.

\section{RELAÇÃO DOS TIPOS}
1) Trichilia augustoi
2) Trichilia barraensis
3) Trichilia catigua

Harms

C.DC

A. Juss. var.

pilosior C.DC
(RB 37923

(RB 18487)

(RB 37921)

\footnotetext{
* Jardim Botânico do Rio de Janeiro e bolsista do CNPG.

* * Convênio IBDF/CETEC

*** Convênio IBDF/CETEC e bolsista do CNPq.
} 
4) Trichilia exce/sa

Benth.

(RB 18489)

5) Trichilia lanceolata

C.DC.

(RB 18491)

6) Trichilia Lecointei

Duke

(RB 8491)

7) Trichilia lobulata

C.DC.

(RB 88283)

8) Trichilia micrantha

Benth

(RB 18493)

9) Trichilia orgaosana

C.DC.

(RB 88284)

10) Trichilia pauloensis

Hoehne

(RB 111205, 31307)

11) Trichilia poeppigii var cinerascens

C.DC.

(RB 20535, 38559)

12) Trichilia pseudostipularis

C.DC var. Sanctae-Catharinae

C.DC.

(RB 88285)

13) Trichilia siqueirae

Ducke

(RB 8304)

14) Trichilia schwackei

C.DC.

(RB 88288)

15) Trichilia tenuiramea

C.DC.

ex. Huber

(RB 20539)

16) Trichilia toledoana

Handro

(RB 111204)

1. Trichilia augustoi Harms (Foto 1)

Harms, Bot.Jarhrb.30(67):34.1902.

"Brasilia: Alto Macahé (Glaziou 17572 - Dec.1888)."

\section{EXEMPLAR RB 37923 - ISÓTIPO}

1. SCHED:

Herb. Mus. Paris

Trichilia augustoi Harms.

Rio J.

Reçu le Glaz. 17572

2. SCHED:

J.B.V. - Jardim Botânico do Rio de Janeiro

n? 37923

Fam. Meliaceae

N. Scient. Trichilia augustoi

Observações: I.B.V. $860-38$.

2. Trichilia barraensis C.DC. (Foto 2)

C. de Candolle in Martius, FI. Bras. 11(1): 222.1878.

“Habitat in silvis prope Barra, munc Manáus, prov. do Alto Amazonas: Spruce n. 1483; ad oram meridionalem flum. Rio Negro usque ad concursum fluminis Solimões: Spruce n. 1417 et 1570!; in silvis udis ad Tagipurú et flumen Amazonum in provincia Pará: Martius! - Apriti m. matus exscit."

19 SCHED:

EXEMPLAR RB 18487 - ISOSINNTIPO

1483

Trichilia?

Prope Barra, Prov. Rio Negro,

Coll. R. Spruce, Apr. 1851. 
Ex- Herb. Musei Britannici

17045

Trichilia?

pr. Barra do Rio Negro

Spruce 1483.

3. SCHED:

Jardim Botânico do $\mathbf{R}$ io de Janeiro

Herbário

n? 18487

Fam. Meliaceae - Data IV - 1851

Nome Scient. Trichilia barraensis C.DC.

Procedência Manáos (Amazonas).

Collegit - Spruce 1483

4. SCHED:

Ex- Herb. Musei Britannici

Trichilia?

17045

Barra do Rio Negro

Spruce 1483

3. Trichilia catigua var. pilosior C.DC. (Foto 3)

C. de Candolle in Martius, FI. Bras. 11(1):211. 1878.

"Habitat in silvis ad Ypanema prov. S. Paulo: Riedel n 2058!; in., Brasilia centrali: Weddell n. 20721; ad Morro de Araraia: Lund!".

EXEMPLAR RB 37921 - ISOSINTIPO

1. SCHED:

Herb. Mus. Paris

Trichilia catigua Juss.

Reçu le Weddell 2072

2. SCHED:

JBV

Jardim Botânico do Rio de Janeiro

n. 37921

Fam. Meliaceae

N. Scient. Trichilia catigua

Observações: JBV 860- 38

4. Trichilia exce/sa Benth. (Foto 4)

Bentham in Hooker, Kew Journ.Bot.3:368.1851

"From the virgin forests, near Santarem" 
19 SCHED:

Trichilia excelsa sp.n.

In vinicibus Santarem, Prov.Para

Coll. R. Spruce

Nov. Mart. 1849-50

2. SCHED:

Jardim Botânico do Rio de Janeiro

N. 18489

Fam. Meliaceae

Nome Scient. Trichilia exce/sa Benth.

Procedencia Santarem (Pará)

Collegit. Spruce 499a

Observação: Encontramos na exsicata os seguintes dizeres:

Ex Herb. Musei Britannici.

\section{SCHED:}

\section{0}

Trichilia excelsa

W. Santarem

$R$ io Amazon. (Spruce)

5. Trichilia lanceolata C.DC. (Foto 5)

C. de Candolle in DC. Monogr. 1:698. 1878

"In Peruvia orientali prope Yurimagas ad flumem Huallago, Majo florescens (Spruce n. 4593, in herb.DC); ad cataractas fluminis Huallaga (Spruce n. 4593, in herb. Kew)."

\section{EXEMPLAR RB 18491 - ISÓTIPO}

19 SCHED:

4593 Moschoxylon

Prope Yurimaguas, ad flumem Huallaga, Peruvia orientalis, coll.

R. Srpuce - Maio 1855.

2. SCHED:

Jardim Botânico do Rio de Janeiro

N. 18491 - data V-1855

Fam. Meliaceae

Nome Scient. Trichilia lanceolata C.DC.

Procedencia Yurimaguas (Peru oriental)

Collegit. Spruce 4593

3. SCHED:

Ex Herb. Musei Britannici

4. SCHED:

19996 - Moschoxylon lanceolata C.DC.

Yurimaguas 
Rio Huallaga

Up. Peru

Spruce 4593

6. Trichilia Le Cointei Ducke (Foto 6)

Ducke, Arch. Jard. Bot. Rio de Janeiro 3:191.1922.

"Habitat in silvis primariis non inundatis circa Obidos, 1.P. Le Cointe 25-8-1916 florifera, n. 16799. "Pracuuba da terra firme" appellatur."

\section{EXEMPLAR RB 8491 - ISÓTIPO}

1. SCHED:

\section{1}

Herb. Amaz. Musei Paraensis (M.Goeldi).

Pará (Brasil)

N. 16799 - Fam. Meliaceae

Trichilia Lecointei Ducke n. sp.

"pracuuba da terra firme"

Localidade: Obidos, matta da t. $\mathrm{a}_{\mathrm{f}}$.

Data 25-8-1916

E? do Pará

Collecionador P. le Cointe.

2. SCHED:

No 8491 - data 25-8-1916

Fam: Meliaceae

Nome scient. Trichilia Le Cointei Ducke

Procedencia Obidos (Pará)

Collegit - P. Le Cointe, Herb. Amaz. 16799.

3. SCHED:

NN 8491 - data 25-8-1916.

Fam. Meliaceae

Nom. Scient. Trichilia Le Cointei Ducke n.sp.

N. vulgar. Pracuuba de terra firme

Procedencia Obidos - matta da terra firme - Pará.

Collegit: P. Le Cointe 16799

7. Trichilia lobulata C.DC. (Foto 7)

C. de Candolle, Bull. Herb. Boiss. Sér. 2(4):364. 1901.

"Minas Gerais ad ripas lacuum prope Rio Nuovo (n. 11824).

\section{EXEMPLAR RB 88283 - ISÓTIPO}

1. SCHED:

Herb. Schwacke 11824

Trichilia lobulata C.DC.

Frutex alt. Testa aurantiaca

Arillus olivaeus. Min. Ger.

19. sept. 95 ad ripas lacuum prope Rio Novo. 
Herb. n? 88283

8. Trichilia micrantha Benth. (Foto 8)

Bentham in Hooker, Kew Journ. 3:369.1851.

"From the Capoeiras, near Barra do Rio Negro".

\section{EXEMPLAR RB 18493 - ISÓTIPO}

19 SCHED:

Trichilia? micrantha sp. $\mathrm{n}$.

In. vinicibus Barra. Prov. Rio Negro, coll. R. Spuce, Dec. - Mart. 1850-51.

2. SCHED:

Jardim Botânico do Rio de Janeiro

N. 18493

Fam. Meliaceae

Nome scient. Trichilia micrantha Benth

Procedencia Manáos (Amazonas)

Collegit Spruce.

3a SCHED:

Ex Herb. Musei Britannici

Observação: $\mathrm{Na}$ exsicata encontravam-se os seguintes dados:

16224, Trichilia? micrantha Benth.

Barra do Rio Negro - Prov. Para (Spruce).

9. Trichilia orgaosana C.DC. (Foto 9)

C. de Candolle, Bull. Herb. Boiss. Sér 2(4):362. 1901.

"Rio de Janeiro, Serra dos Orgaos (n. 4354)."

\section{EXEMPLAR RB 88284 - ISÓTIPO}

1. SCHED:

Herb. Schwacke n? 4354

Trichilia orgaosana C.DC. sp. $\mathrm{n}$.

7-1-1883 - Serra dos Orgấos

2. SCHED:

Herb. $n$ ? 88284

Fam. Meliaceae

Trichilia orgaosana C.DC.

Proced. Srrra dos Orgãos

Obs. Herb. Schwacke 4354

Data 7-1-1883 
10. Trichilia pauloensis Hoehne (Fotos 10,11)

Hoehne, Ostenia 301.1933.

"Muitos exemplares vivos do Jardim Botânico do Estado de S. Paulo, marcados com o n. 107 e material exsiccado das mesmas, $n$ ? 28.348 do nosso herbário colletado em Outubro e Novembro de 1931".

\section{EXEMPLAR RB 111205 - ISÓTIPO}

Inst. de Botanica

S. Paulo - Brasil - Herb. n. 28348

Trichilia pauloensis Hoehne

Isotypus

Brasil - Estado de São Paulo: S. Paulo,

nativa na matta do Parque do Estado e Jardim Botânico,

fI. X-XI-1931.

Árvore pequena com flores amareladas.

Coll. F. C. Hohne - n?

\section{EXEMPLAR RB 31307 - ISÓTIPO}

1. SCHED:

1 Seção Botanica e Agronomia do Instituto Biológico de defesa agricola e animal.

No 28348 - Meliaceae

Trichilia pauloensis Hoehne

"Catigua Pequeno"

Jardim Botânico, S. Paulo, 28-9-1931

Planta viva n? 107 - Leg. - Det. F.C. Hoehne

2. SCHED:

Herbário do Jardim Botânico do Rio de Janeiro

Registro n? 31307

11. Trichilia poeppigii var.cinerascens C.DC. (Fotos 12,13)

C. de Candolle, Bull. Herb. Boiss. ser. 2 (6): 985.1906.

“Purus. Bon Lugar, in silva, julio florens (J.Huber n. 3949 in h. Mus. Goeldi h. Cand.); Alto Purus, Ponto Alegre, in silva (n. 4410, 4518 ibid); Rio Purus, Monte Verde, terr. firm., aprilli maturescens (n. 4576 ibid.)."

19 SCHED:

1.9 EXEMPLAR RB 20535 - ISOSINTIPO

H.A. 4576

Trich. Poeppigii C.DC.

var. cinerescens C. DC.

Monte Verde, R. Purus, terra firme

28-4-1904 J.Huber.

2. SCHED:

Jardim Botanico Rio de Janeiro

n. 20535 - Data 28-4-1904 


\section{Fam. Meliaceae}

Nome Scient. Trichilia Poeppigii C.DC.

var. cinerescens C.DC.

Procedencia Monte Verde, Alto Purus (Amazonas)

Collegit. J. Huber, Herb. Amaz. 4576

Determ. C. de Candolle

$$
\text { 2. EXEMPLAR RB } 38559 \text { - ISOSINTIPO }
$$

\section{SCHED:}

\section{H.A. 3949}

Trich. Poeppigii C.DC. var cinerescens C.DC.

Bom logus, Purús, matta, 19-7-1903, A. Goeldi

\section{SCHED:}

Jardim Botânico do Rio de Janeiro

N. 38559 - Data 19-7-1903

Fam. Meliaceae

Nome Scient. Trichilia Poeppigii C.DC.

var. cinerescens C. DC.

Procedencia: Bom lugar, Purús (Amazonas).

Collegit A. Goeldi, Herb. Amaz. 3949.

12. Trichilia pseudostipularis var. sanctae - catharinae C.DC. (Foto 14)

C. de Candolle, Bull. Herb. Boiss. Sér 2(4):362. 1901.

"Santa Catharina in silva virginea ad fluem I tapócu (n.13012)."

$$
\text { EXEMPLAR RB } 88285 \text { - ISOTIPO }
$$

\section{SCHED:}

Herb. Schwacke 13012

Trichilia pseudostipularis C.DC. Sanctae Catharinae C.DC. var. nov.

Frutex hum. alt.

Sanctae Catharina C.DC var. nov.

4-IX-97 in sylv. ad fl. I tapocú.

2. SCHED:

Herb. n? 88285

Meliaceae

Trichilia pseudostipularis C.DC.

Proced. Santa Catarina

Herb. Schwacke 13012

Data 4-1X-97

\section{Trichilia Siqueirae Ducke (Foto 15)}

Ducke, Arch. Jard. Bot. Rio de Janeiro 3:192.1922.

"Habitat in silvis primaris non inundatis ad stationem Peixeboi viae ferreae inter Belém at Bragança 1.R. Siqueira 15-7-1907 florif., n. 8288." 
Jardim Botanico do Rio de Janeiro

N. 8304 data 15-7-907

Nome scient. Trichilia Siqueirae Ducke n.sp.

Procedencia - Peixe-boi (Belém- Bragança)

Collegit. A.Siqueira, Herb. Amaz. Mus. Pará 8288.

2. SCHED:

15-7-1907

Areuna - Areuna

R. Siqueira - Peixe boi.

14. Trichilia schwackei C.DC. (Foto 16)

C. de Candolle, Bull. Herb. Boiss. Sér 2 (4): 363. 1901.

"Rio de Janeiro, Serra da Bica prope Cascadura (n. 5150)."

\section{EXEMPLAR RB 88288 - ISOTIPO}

1. SCHED:

Meliaceae

Herb. Schwacke n: 5150

Trichilia schwackei C.D.C. sp.nov.

Rio de Janeiro

26-Aug.1886. Serra da Bica.

2. SCHED:

Herb. NO 88288

Fam. Meliaceae

Trichilia schwackei C.DC.

Proc. Rio de Janeiro - Serra da Bica.

Obs. Herb. Schwacke n. 5150

Data 26-8-1886

15. Trichilia tenuiramea C.DC. ex Huber (Foto 17)

Huber, Bol. Mus. Goeldi 5 (7): 436. 1909.

"Castanhaes do Rio Cumina mirim, in silvis, Decembri (A.Ducke n. 7944 in h.Mus.Goeldi, h.Cand.)."

\section{EXEMPLAR RB 20539 - ISOTIPO}

19. SCHED:

H.A. 7944

Trichilia tenuiramea C.DC. n.sp.

Castanhaes do Cuminá-mirim.

12-12-1906 A.D. 
SCHED:

N. 20539

Fam. Meliaceae

Nom. Scient. Trichilia tenuiramea

Procedencia Castanhaes do Rio Cuminá-mirim (Trombetas: Pará).

Collegit. A. Ducke, Herb.Amaz. 7944

Determ. por C. de Candolle

\section{Trichilia toledoana Handro (Foto 18)}

Handro, Arq. Bot. Est. S. Paulo 3: 224. 1962.

“typus: M.Kuhlmann 4469

"Material estudado: Brasil, Estado de S.Paulo nativa no Jardim Botânico, fruct. immat. 9 - VII-1950 e capsulas velhas sem sementes (capsulae veteres sine semen) XII/1950, col.M.Kuhlmann 2541 (SP 47019); idem, fl. 28-XI-1958, col. M.KuhImann 4469 (SP 56506 Holotypus).

\section{EXEMPLAR RB 111204 - ISOTIPO}

\section{SCHED:}

Instituto de Bot. de S.Paulo - Herb. n. 56506

Trichilia toledoana Handro - Isotypus

Brasil - Estado de São Paulo: São Paulo

nativa no Jardim Botânico, 28- XI- 1958. Arvore da mata, de cerca

de $15 \mathrm{~m}$ de altura. Flores aromáticas com corola alvacenta.

Col. Moyses Kuhlmann - n? 4469

\section{ABSTRACT}

This paper continues the survey of the types from the Rio de Janeiro Botanical Garden Herbarium, (RB), Meliaceae I, following the same criterion as the former. Photographs illustrate each species cited by the author.

\section{AGRADECIMENTOS:}

Ao Conselho Nacional de Desenvolvimento Científico e Tecnológico (CNPq) pelas Bolsas
Concedidas. Concedidas.

\section{BIBLIOGRAFIA}

BENTHAN, M.G. et J.D. HOODER - 1851. Report on the plants collected by Mr. Spruce in Brazil in Kook Journ Bot 3: 368 - 369

CANDOLLE, C. - 1878. MELIACEAE in Martius FI. Bras. 11 (1): 165-228. tab. 50-65. 
CANDOLLE, C. - 1878. Meliaceae in Candolle - Monog. Phan. 1: 399-752, tab. 6-9.

CANDOLLE, C. - 1901. Piperaceae et Meliaceae Brasiliensis - Bull. Herb. Boisa ser. 2 (4): 353-366.

CANDOLLE, C. - 1906. Meliaceae novas vel iterum lectae et Rutacea Nova Bull. Herb. Boiss. ser. 2,6 (12): 64-985.

DUCKE, A. - 1922. Plantas nouvelles ou peu connues de la región amazonienne in Arch. Jard. Bot. Rio de Janeiro 3: 3-269, 27 est

GUIMARÃES, E.F. et J.G. PEREIRA - 1965. Typus do Herbário do Jardim Botânico do Rio de Janeiro II - Arq. Jard. Bot. Rio de Janeiro 18. 261-267.

GUIMARÃES, E.F. - 1966. Typus do Herbário do Jardim Botânico do Rio de Janeiro IV Rodriguésia 25 (37): 239-264.

HANDRO, O - 1962. Plantas novas - Notas sobre algumas outras já conhecidas da Flora do Brasil. Arq. Bot. Est. S. Paulo 3 (5): 219-236.

HARMS, H. - 1902. Meliaceae in Bot. Jahr. 30 :67): 32-35

HOEHNE, F.C. - 1933. Observações e quatro novas espécies arborescentes do incipiente Jardim Botânico do Estado de São Paulo - Ostenia 287 - 304. 8 est.

HUBER, J. - 1909. Materiais para a Flora Amazônica VII - Plantas Duckeaneae austro guyanensis. Bol. Mus. Goeldi 5 (7): 294-436.

MENDES MARQUES, C.M. et A.E. MONTALVO - 1976. Levantamento dos tipos do Herbário do Jardim Botânico do Rio de Janeiro (Bignoniaceae II) Rodriguésia 28- (41): 37-59, 9 pranchas.

OCCHIONI, P. - 1949. Lista dos "Typus" do Herbário do Jardim Botânico do Rio de Janeiro. Lilloa, Tucuman, 27: 419-401.

OCCHIONI, P. - 1952. Lista dos "Typus" do Herbário do Jardim Botânico do Rio de Janeiro II. Dusenia, $3(4): 251-262$.

OCCHIONI, P. - 1953. Lista dos "Typus" do Herbário do Jardim Botânico do Rio de Janeiro. Tribuna Farmacêutica, Curitiba - (21 (10): 163-165.

RIBEIRO DE SOUZA, F.A. et L.C. ABREU BENEVIDES - 1977. Levantamento dos Tipos do Herbário do Jardim Botânico do Rio de Janeiro (Leguminosae Caesalpinoideae - II). Arq. Jard. Bot. Rio de Janeiro, 20-93-116, 22 pranchas. 

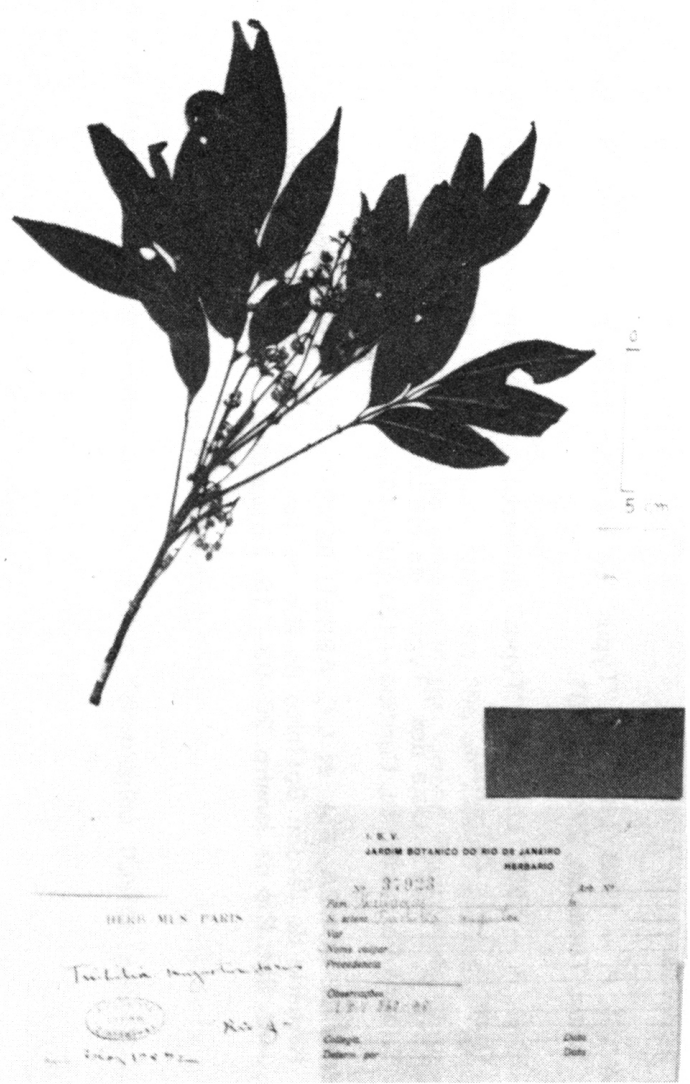

FOTO 1: Trichilia augustoi Harms
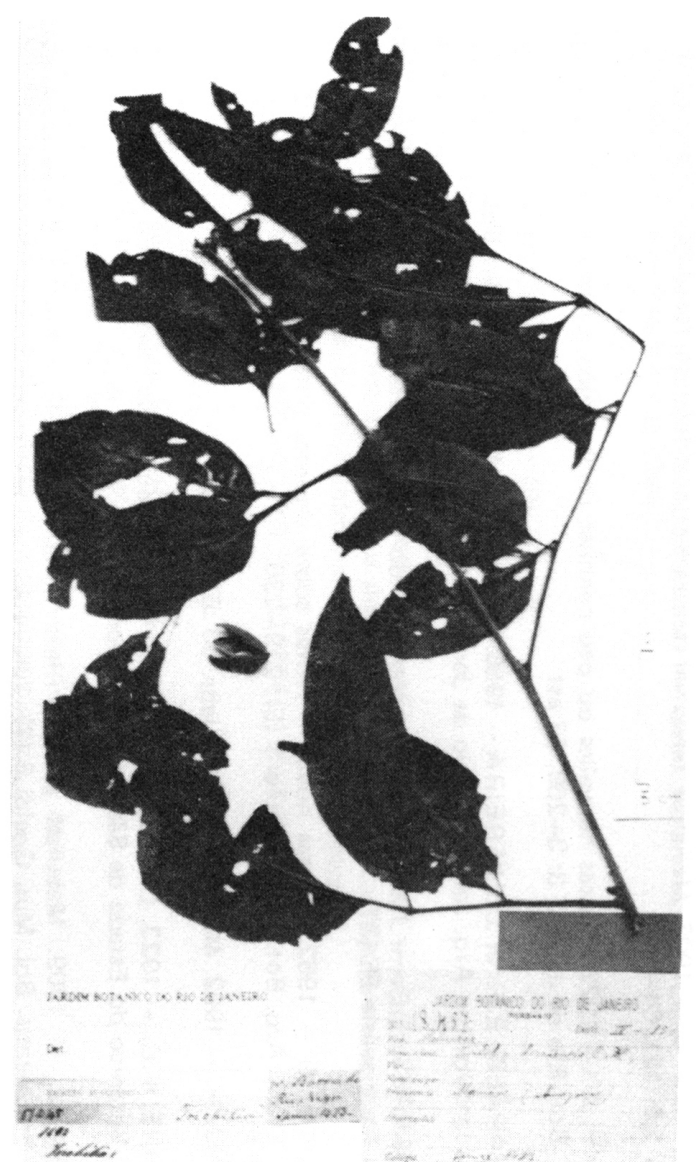

FOTO 2: Trichilia barraensis C. DC. 

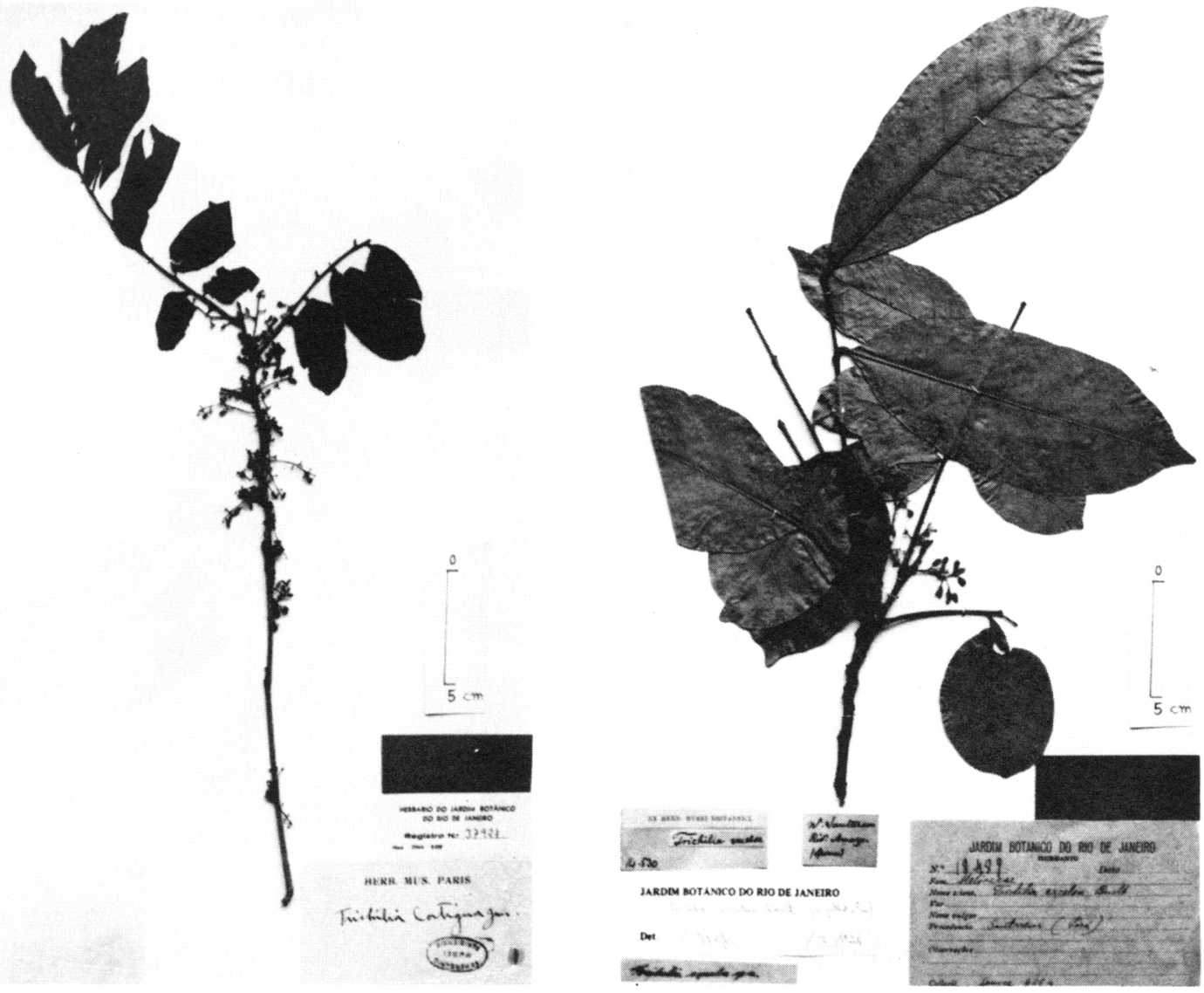

FOTO 3: Trichilia catigua A. Juss.

FOTO 4: Trichilia exce/sa Benth 


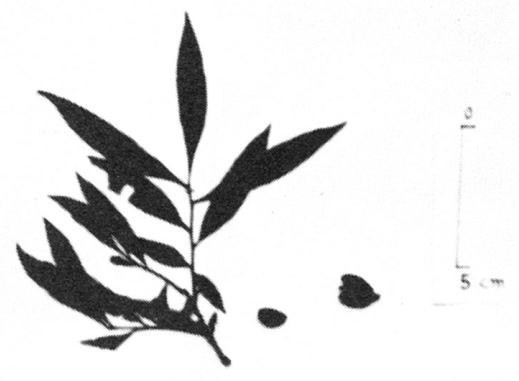

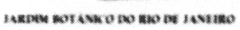

an
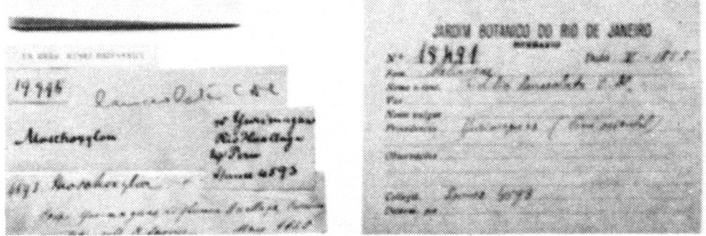

FOTO 5: Trichilia lanceolata C. DC.

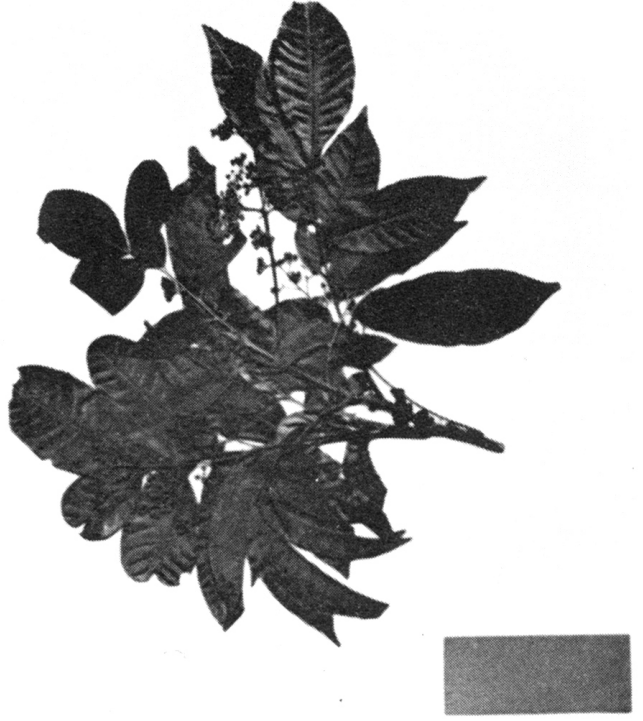

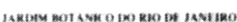

int

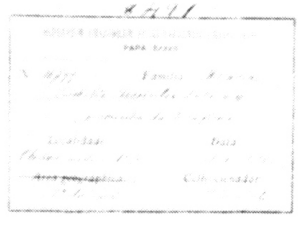

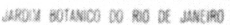

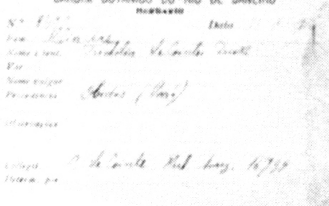

FOTO 6: Trichilia LeCointei Ducke 


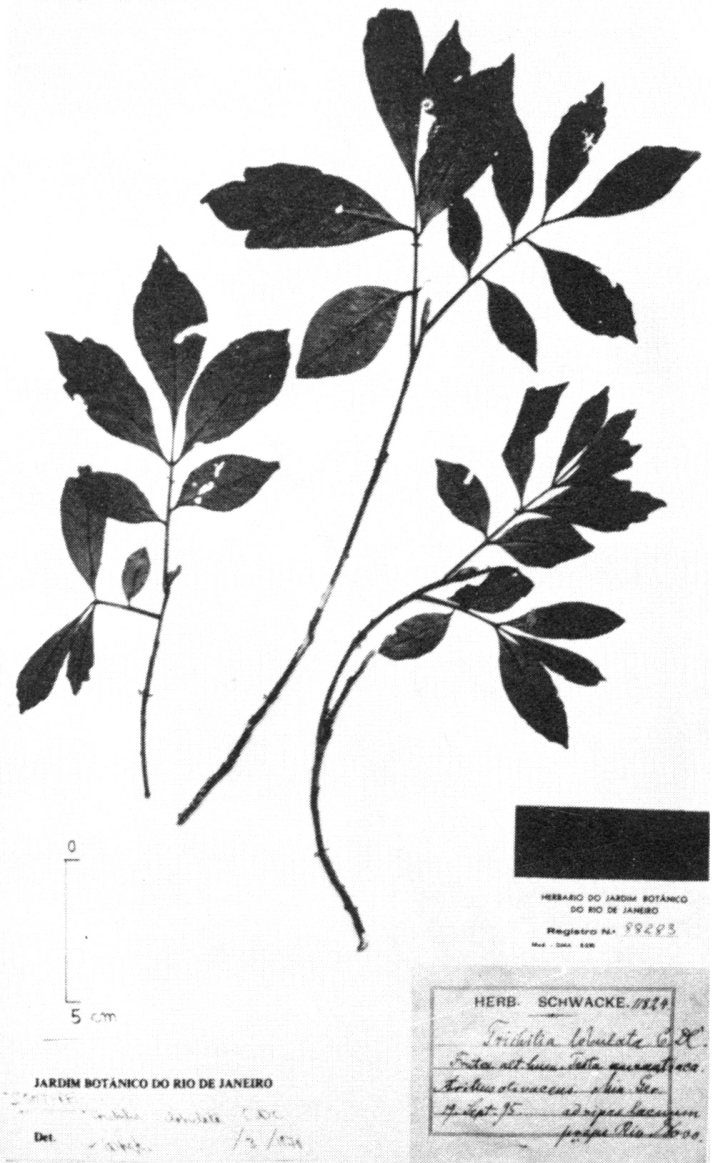

FOTO 7: Trichilia lobulata C. DC

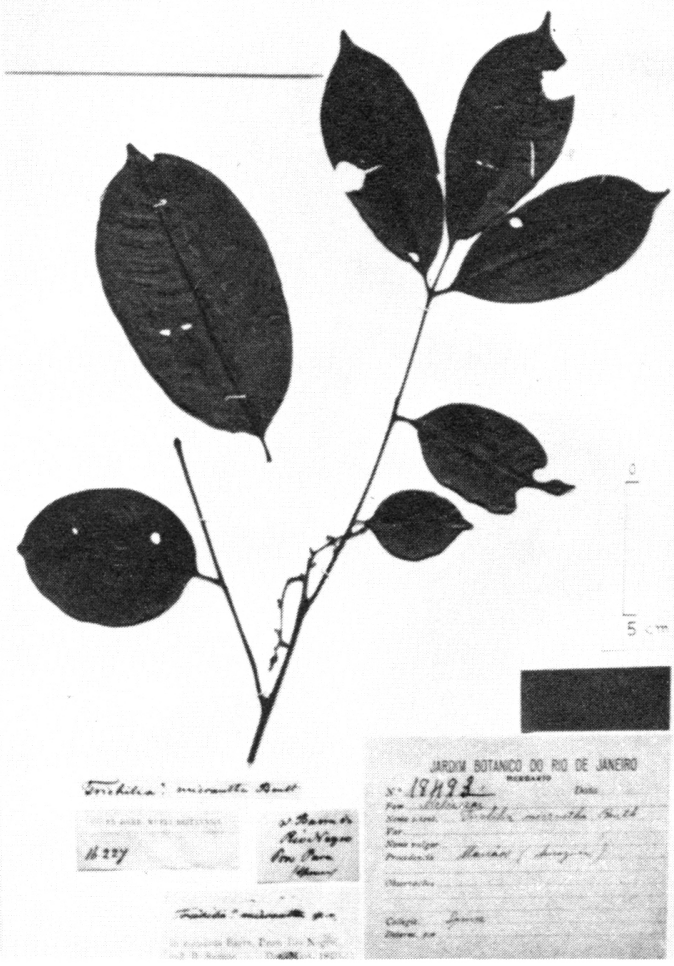

FOTO 8: Trichilia micrantha Benth 


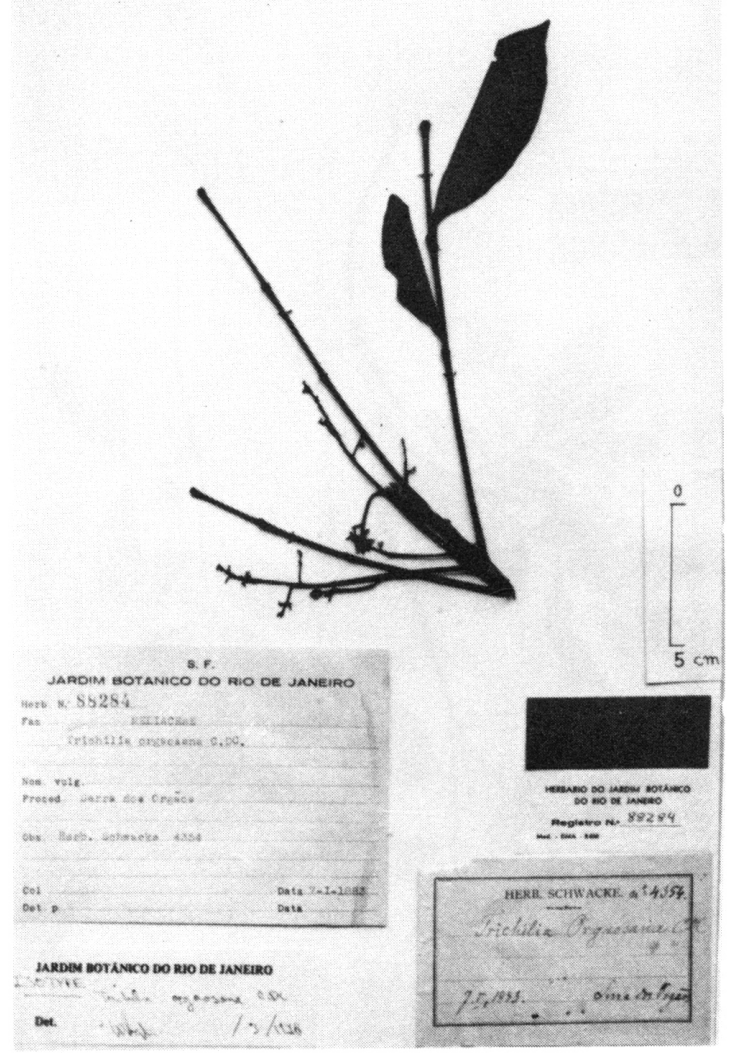

FOTO 9: Trichilia orgaosana C. DC.

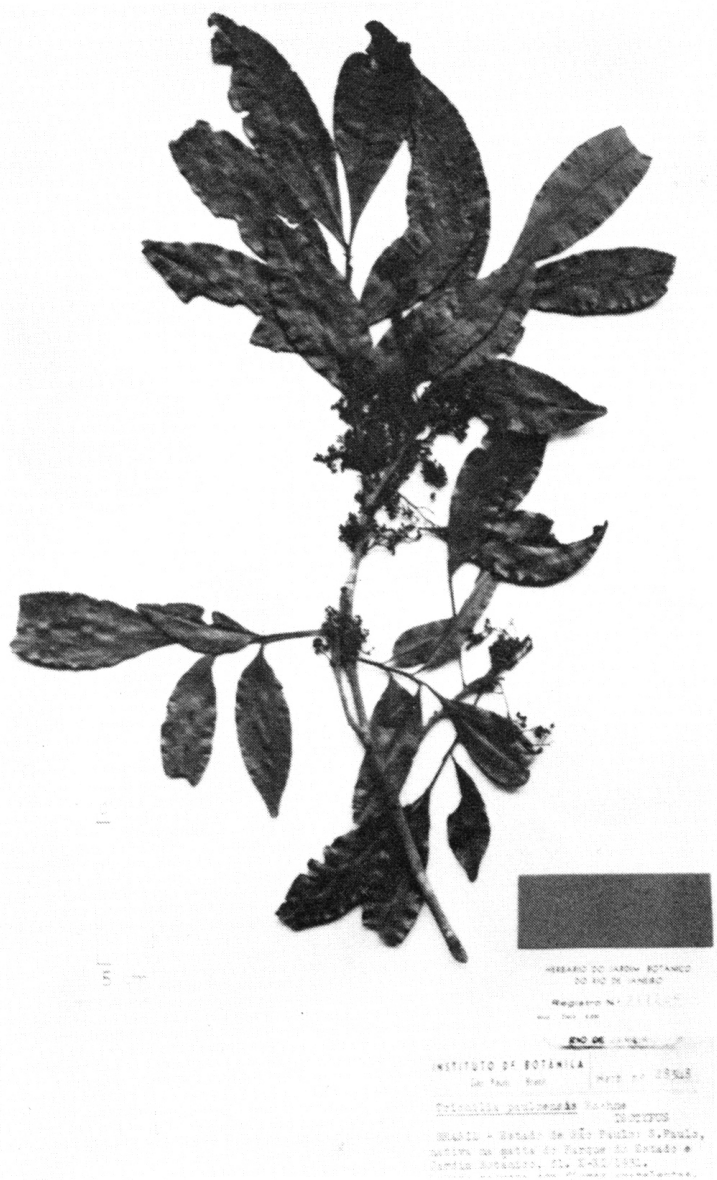

FOTO 10: Trichilia pauloensis Hoehne 


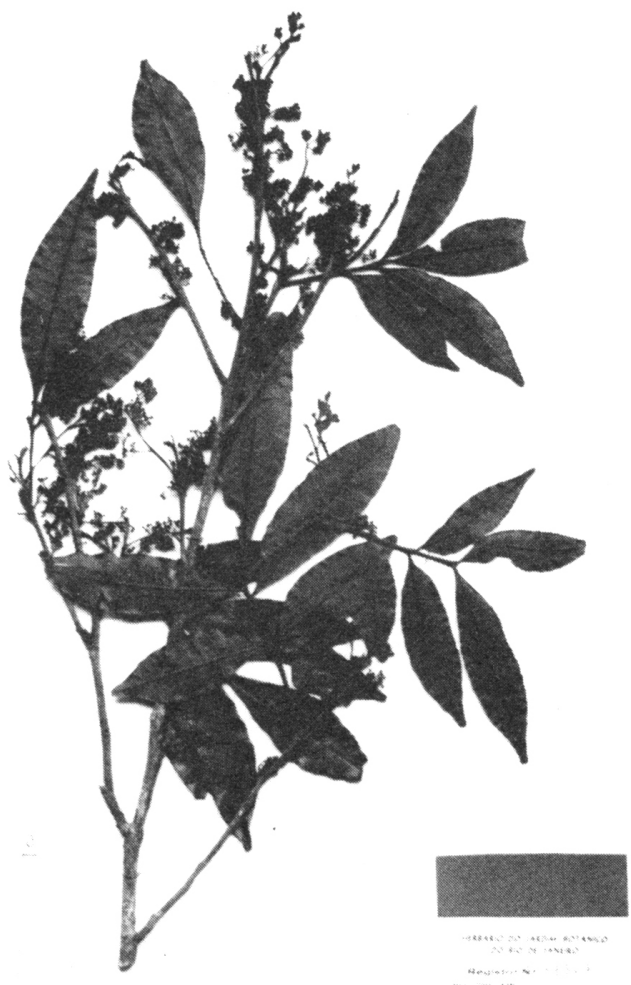

FOTO 11: Trichilia pauloensis Hoehne

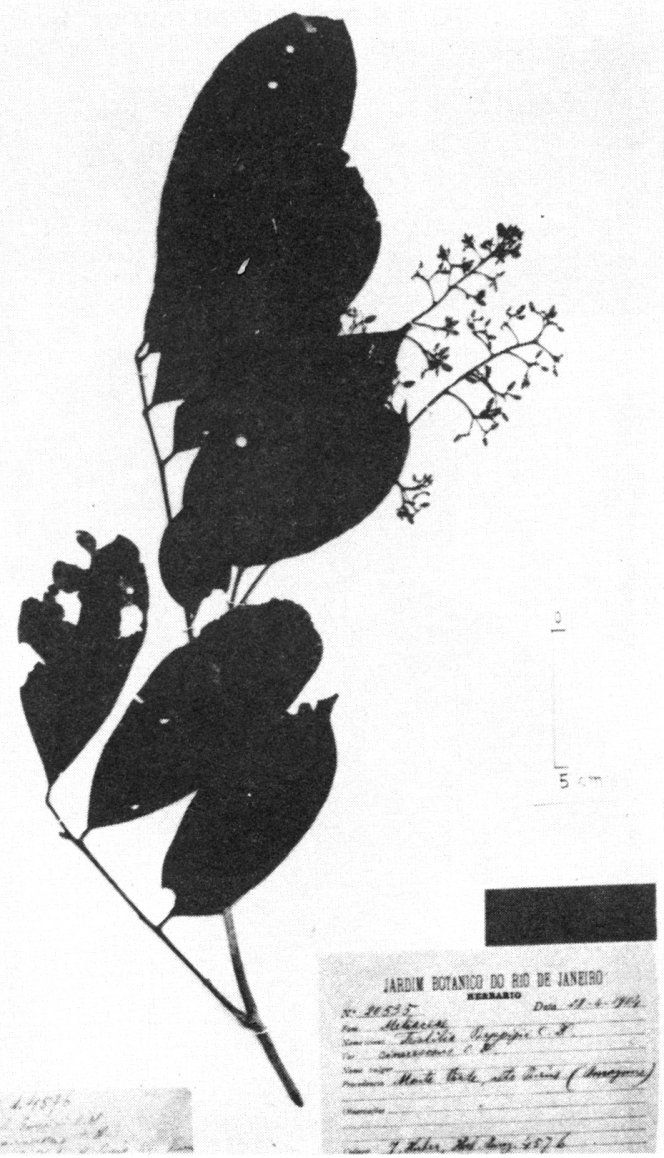

FOTO 12: Trichilia poeppigii C. DC. - var. cinerescens C. DC. 


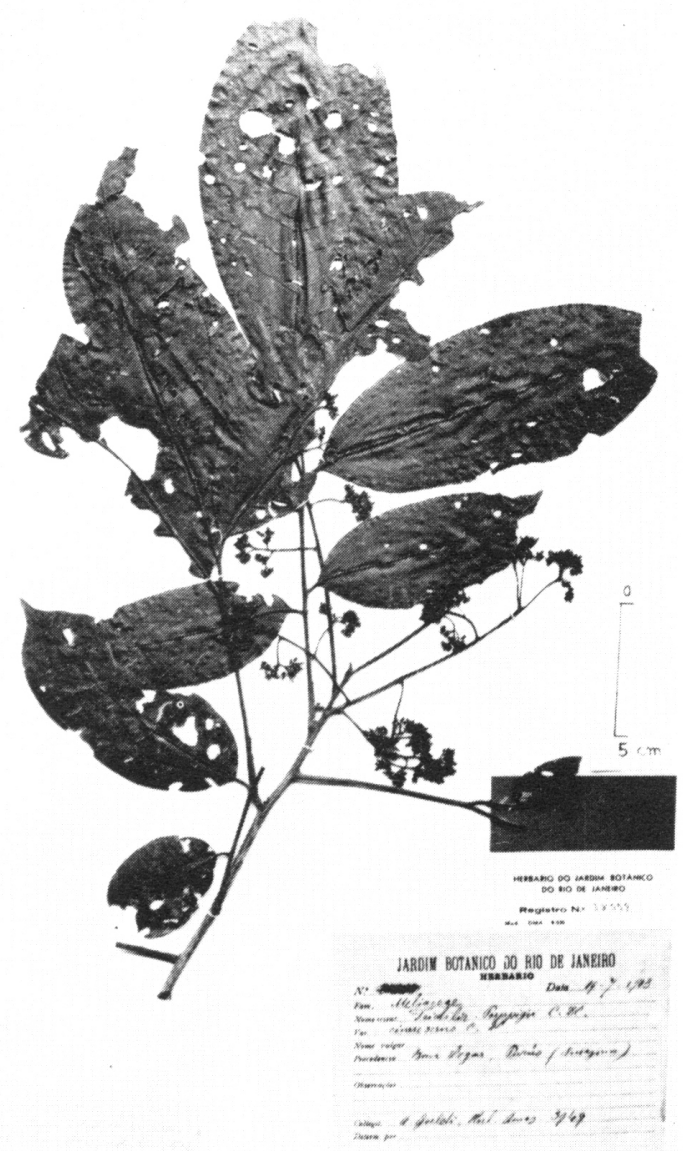

FOTO 13: Trichilia poeppigii C. DC. var. Cinerescens

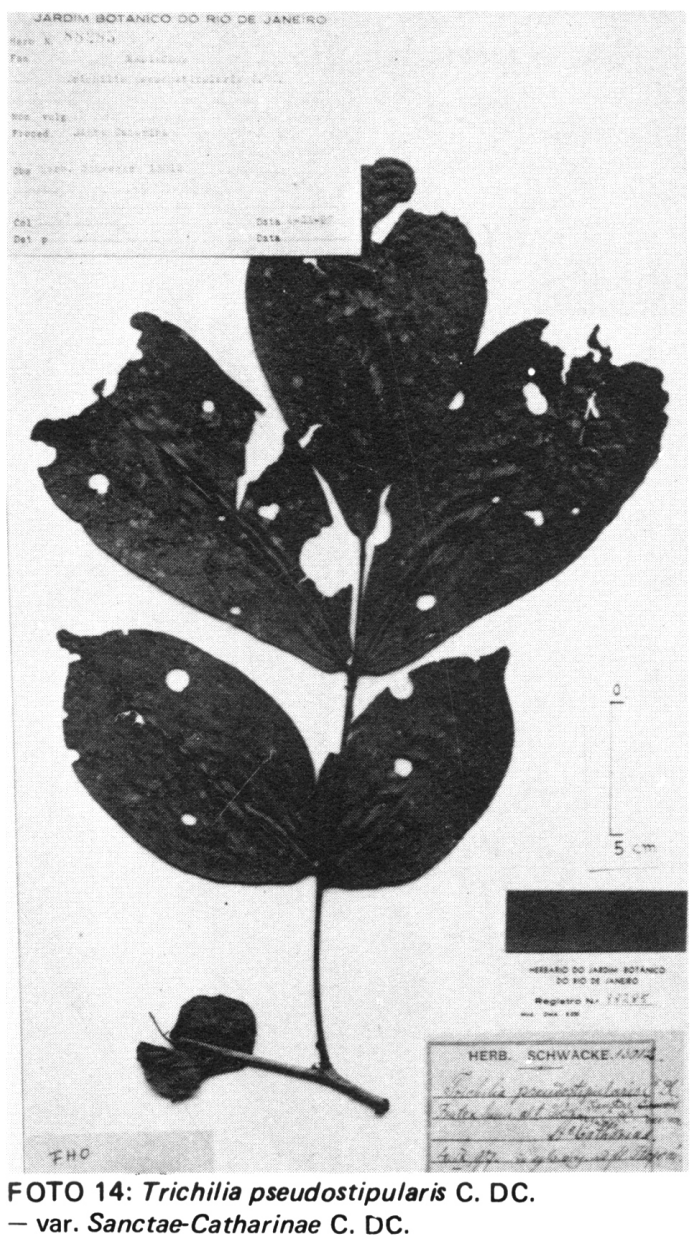




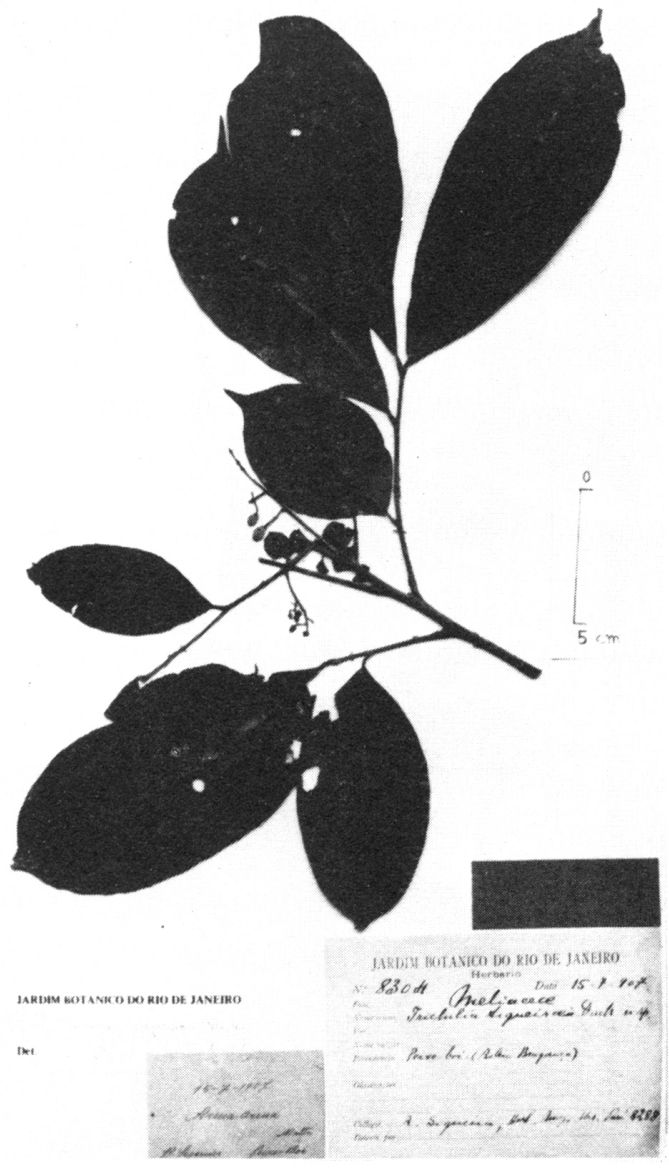

FOTO 15: Trichilia siqueirae Ducke

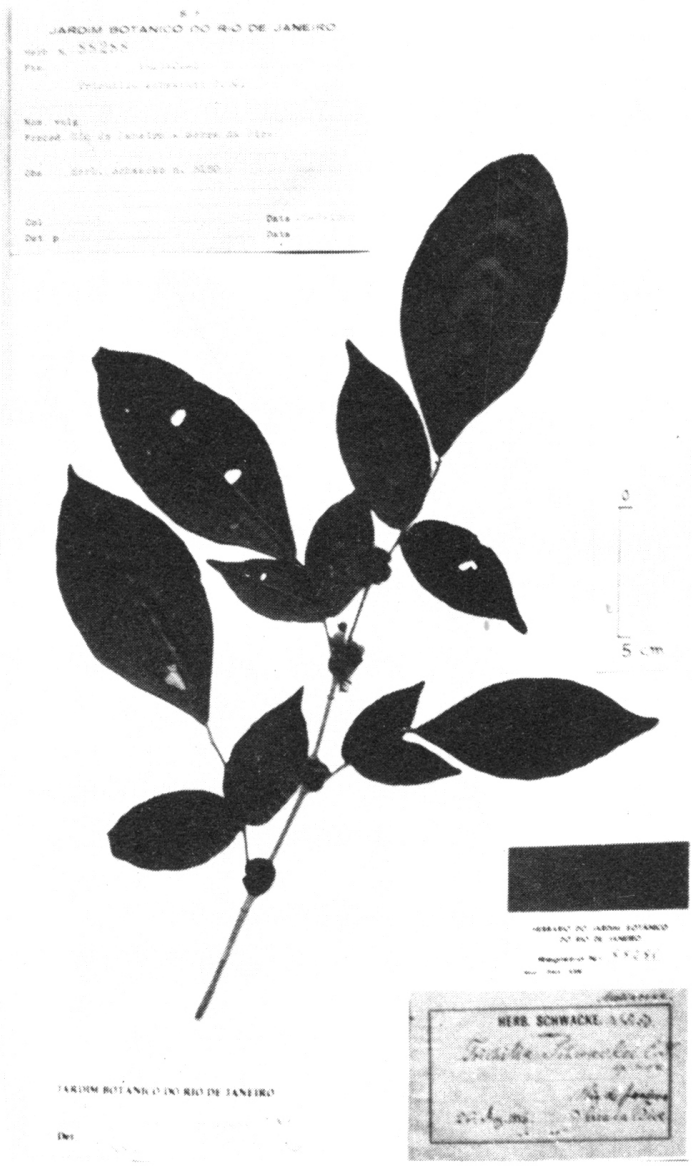

FOTO 16: Trichilia schwackei C. DC. 

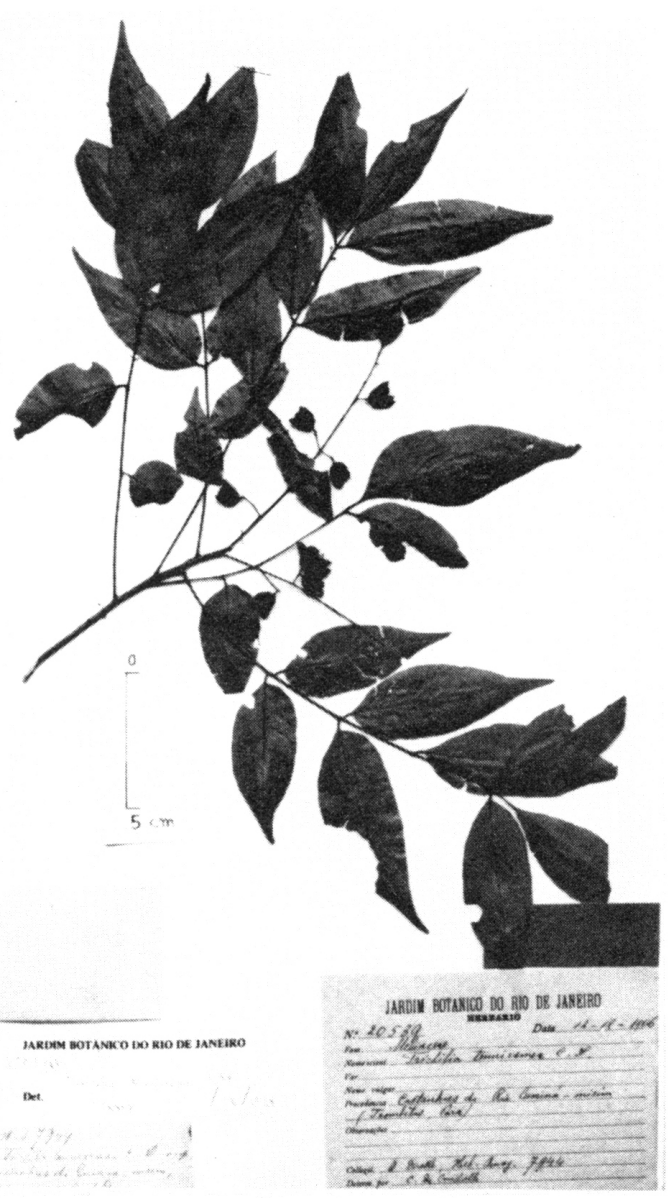

FOTO 17: Trichilia tenuiramea C. DC.

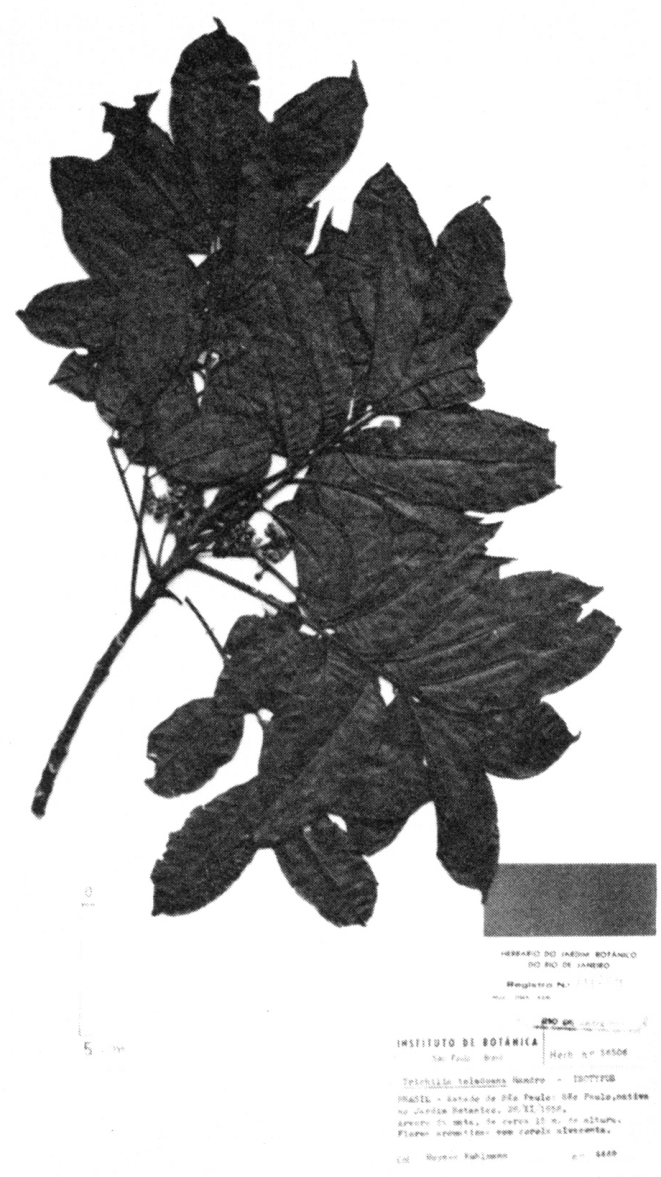

FOTO 18: Trichilia toledoana Handro 